\title{
Is short-term antiarrhythmic therapy an option?
}

S hort-term antiarrhythmic drug therapy with flecainide can prevent most episodes of recurrent atrial fibrillation $(\mathrm{AF})$ in patients who have undergone electrical cardioversion. Despite being not as effective as long-term therapy, a short duration of treatment could be the ideal strategy for patients who are at increased risk of drug-related complications, or at low risk of AF recurrence. These findings, from the Flecainide Short-Long (Flec-SL) trial, have been reported by Paulus Kirchhof and colleagues in the Lancet.

To the investigators' knowledge, Flec-SL was the first comparison of short-term and long-term antiarrhythmic drug therapy after cardioversion in patients with AF. The results, therefore, have important implications for clinical practice.

Flecainide is an antiarrhythmic agent that has been in clinical use since the 1980s and mainly inhibits the cardiac sodium channel. This drug prevents recurrent AF by prolonging the duration of the atrial action potential, thereby ameliorating the effects of electrical remodeling caused by AF. Recovery from electrical remodeling with normalization of action-potential duration often occurs once sinus rhythm has been restored for a few weeks. A short course of therapy with an antiarrhythmic agent could, therefore, give the atria a chance to 'heal' itself and stop the vicious circle of AF begetting AF. In addition, "antiarrhythmic drug therapy is limited by rare, but potentially hazardous, side effects," explains Dr Kirchhof. "Hence, a shortening of therapy duration would be desirable to reduce such outcomes."

The Flec-SL trial was conducted between May 2007 and March 2010 at 44 centers in the German Atrial Fibrillation Competence Network. The study was prospective, randomized, and openlabel with blinded end-point assessment. A total of 635 patients aged $>18$ years, with persistent $\mathrm{AF}$ and an indication for cardioversion were enrolled in the trial. All patients received $\geq 48 \mathrm{~h}$ of treatment

\author{
4 ... our data demonstrate that \\ electrical remodeling ... is not \\ the only cause of recurrent AF \\ after cardioversion... 77
}

with flecainide before cardioversion. They were then randomly assigned in a 1:1:1 ratio to either no further antiarrhythmic drug treatment, short-term flecainide therapy ( 4 weeks), or long-term flecainide therapy ( 6 months). In both flecainide groups, the initial dose was $100 \mathrm{mg}$ twice per day, but could be uptitrated to $100 \mathrm{mg}$ three times per day if necessary. Patients were followed up for 6 months with daily electrocardiography and $24 \mathrm{~h}$ Holter monitoring if AF recurrence was suspected. Quality of life was also assessed, using the SF- $12^{\circledast}$ (Medical Outcomes Trust, Inc., Waltham, MA, USA) questionnaire and the Karnofsky score.

An initial analysis was performed in the first 242 patients 4 weeks after treatment commenced. Flecainide therapy (both groups) was found to be superior to no drug therapy (survival free from AF: $70.2 \%$ versus $52.5 \% ; P=0.0160$ ). For the remainder of the trial, only short-term and long-term therapy were assessed. After 6 months of follow-up, more patients receiving short-term therapy had developed recurrent $\mathrm{AF}$ than those in the long-tem therapy group ( $46 \%$ versus $39 \%$; difference in event-free survival $7.9 \%$, $P=0.2081$ for noninferiority). Therefore, noninferiority of short-term treatment was not proven. Long-term treatment was shown to be the superior strategy in a post hoc landmark analysis (difference in eventfree survival $14.3 \%, P=0.0001$ ). However, no differences were reported between the short-term and long-term groups in quality-of-life scores, number of hospital admissions for $\mathrm{AF}$, or left ventricular function at 6 months.

No deaths occurred in any of the treatment groups. Serious adverse cardiovascular or cerebrovascular events

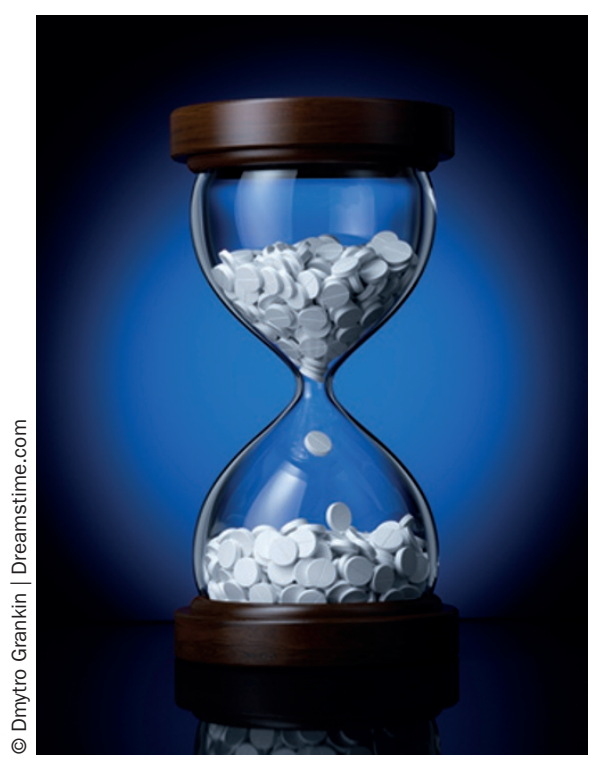

occurred in only $3 \%$ of the total study population, with no differences between short-term and long-term therapy.

"Our data demonstrate that electrical remodeling, while playing an important contribution ... is not the only cause of recurrent AF after cardioversion," says Dr Kirchhof. "We believe that other factors that cause recurrent AF in the long term ... should be studied and characterized at the mechanistic level to develop new, additional rhythm-control therapies in AF." In a Lancet Editorial, A. John Camm and Irene Savelieva (St George's University of London, UK) postulate that the findings of the Flec-SL trial could be extrapolated to other antiarrhythmic drugs, with the exception of amiodarone owing to the long biological half-life (several months) of this drug that prevents its use for short-term treatment.

\section{Alexandra King}

